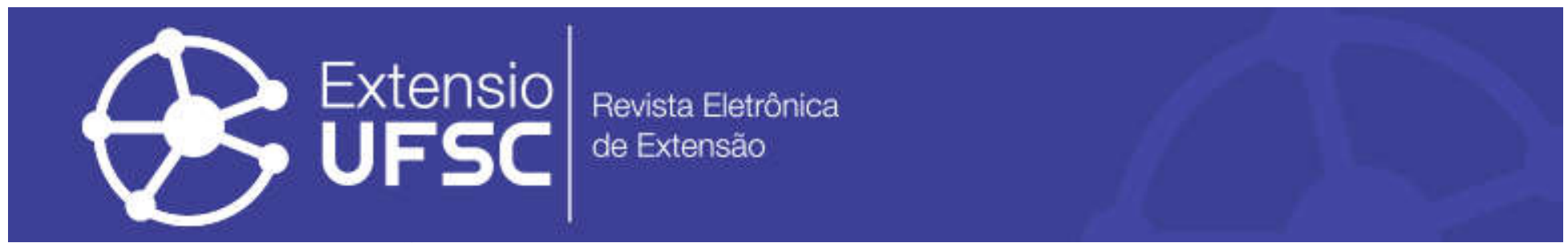

\title{
A ATUAÇÃO DA ODONTOLOGIA HOSPITALAR EM UMA UNIDADE CARDIOVASCULAR INTENSIVA
}

\author{
Orlando Luiz do Amaral Júnior \\ Universidade Federal de Santa Maria \\ orlandodoamaraljr@gmail.com \\ Maitê Munhoz Scherer \\ Universidade Federal do Rio Grande do Sul \\ maitemunhozscherer@hotmail.com
}

\author{
Paolla Zellya Borges \\ Universidade Federal de Santa Maria \\ paolla.borges@gmail.com \\ Alexsandra da Silva Botezeli Stolz \\ Universidade Federal de Santa Maria \\ alebotzel@gmail.com
}

\section{Resumo}

A intervenção odontológica em pacientes hospitalizados é necessária tanto para evitar o desenvolvimento de doenças sistêmicas, como para diminuir as consequências daquelas já instaladas, resultando em um relacionamento direto entre o cirurgião-dentista, paciente, equipe multiprofissional e a instituição hospitalar. O objetivo deste artigo é apresentar a atuação de uma equipe odontológica em Unidade de Cardiologia Intensiva (UCI); uma subunidade de uma Unidade de Terapia Intensiva (UTI) de um Hospital Universitário. Um total de 312 pacientes receberam acompanhamento odontológico no período em que estiveram internados entre janeiro de 2015 e dezembro 2017. A maioria dos pacientes que foram atendidos na UCI tinha idade superior a 60 anos. Este texto enfatiza a importância da presença de Cirurgiões Dentistas em ambiente hospitalar, considerando que há uma série de ações preventivas, diagnósticas, terapêuticas e paliativas em saúde bucal que podem influenciar a saúde sistêmica e a qualidade de vida dos pacientes. Em suma, a atenção odontológica favorece a atenção integral ao paciente, sendo fundamental para a prevenção de agravos, melhorando sintomas que causam desconforto e afetam o bem-estar do paciente.

Palavras-chaves: Odontologia Hospitalar. Saúde Bucal. Assistência Integral à Saúde.

\section{THE ACTION OF HOSPITAL DENTISTRY IN A CARDIOVASCULAR UNIT}

\begin{abstract}
Dental intervention in hospitalized patients is necessary both to prevent the development of systemic diseases and to reduce the consequences of those already installed, resulting in a direct relationship between the dentist, patient, multidisciplinary team and the hospital. The purpose of this study is to present the experience of the performance of a dental team in an Intensive Cardiology Unit (ICU); a subunit of an Intensive Care Unit (ICU) of a University Hospital.312 patients who received dental care during the period they were hospitalized between January 2015 and December 2017.Most of the patients who were seen at the ICU were older than 60 years. This article emphasizes the importance of the presence of Dental Surgeons in a hospital environment, considering that there are a series of preventive, diagnostic, therapeutic and palliative actions in oral health that can influence the systemic health and quality of life of patients. Dental care favors comprehensive patient care, being essential in preventing injuries and improving symptoms that cause discomfort and affect the patient's well-being.
\end{abstract}

Keywords: Hospital Dentistry. Oral Health. Comprehensive Health Care.

\section{LA ACCIÓN DE LA ODONTOLOGÍA HOSPITALARIA EN UNA UNIDAD CARDIOVASCULAR INTENSIVA}

\section{Resumen}

La intervención dental en pacientes hospitalizados es necesaria tanto para prevenir el desarrollo de enfermedades sistémicas como para reducir las consecuencias de las ya instaladas, lo que resulta en una relación directa entre el dentista, el paciente, el equipo multidisciplinario y el hospital.El propósito de este estúdio es informar el desempeño de un equipo dental en una Unidad de Cardiología Intensiva (UCI); Una subunidad de una Unidad de terapia intensiva (UTI) de un Hospital Universitario.312 pacientes que recibieron atención dental durante el período en que fueron hospitalizados entre enero de 2015 y diciembre de 2017. La mayoría de los pacientes que fue atendida em la UCI era mayor de 60 años. Este artículo enfatiza la importancia de la presencia de cirujanos dentales em un entorno hospitalario, considerando que hay una serie de acciones preventivas, diagnósticas, terapéuticas y paliativas enlasalud oral que pueden influir em la salud sistémica y lacalidad de vida de los pacientes. Em resumen, la atención dental favorece la atención integral del paciente, siendo esencial para prevenir lesiones y mejorar los síntomas que causan molestias y afectan el bien estar del paciente.

Palabras clave: Odontología Hospitalaria. Salud Bucal. Atención Integral de Salud. 
A atuação da odontologia hospitalar em uma unidade cardiovascular intensiva

\section{INTRODUÇÃO}

Em unidades hospitalares, é importante que o monitoramento dos diferentes órgãos e sistemas que não são considerados as causas diretas das doenças de base do paciente não seja deixado de lado. Essa atenção evita um maior comprometimento da saúde geral do paciente, contribuindo para um prognóstico mais favorável do caso (COLL et al., 2020). O acúmulo de micro-organismos na superfície dos dentes e cavidade bucal em pacientes internados em Unidades de Terapia Intensiva (UTI) gera um aumento significativo no tempo de internação, ocorrendo um aumento de patógenos respiratórios que colonizam o biofilme bucal (GOMES; ESTEVES, 2012a).Entre as consequências da presença desses patógenos, destacam-se a formação de placa dental e a bronquiaspiração como fatores importantes para o desenvolvimento de pneumonia, uma vez que a traqueia e os pulmões podem ser colonizados por microrganismos provenientes desses sítios (TERPENNING, 2005).

Os patógenos respiratórios que causam pneumonias estabelecidos no biofilme são difíceis de serem removidos, pois este propicia uma proteção às bactérias, tornando-as mais resistentes aos antibióticos (GOMES; ESTEVES, 2012a). Sendo assim, a descontaminação da boca se torna de extrema importância na prevenção das pneumonias aspirativas em pacientes internados em UTI (TAKAHAMA JR et al., 2020).

Com a preocupação de estabelecer a melhor forma de intervenção nos ambientes hospitalares, diversas pesquisas vêm sendo realizadas com o intuito de avaliar os efeitos do digluconato de clorexidina $0,12 \%$ no biofilme dental e na infecção gengival. Os resultados mostram-se positivos em relação à redução do acúmulo de placa, à diminuição do sangramento gengival e à diminuição da colonização de diversos tipos bacterianos causadores de pneumonia (AMARAL; CORTÊS; PIRES, 2009).Assim, o uso da clorexidina na higiene oral em pacientes sob ventilação mecânica pode influenciar na redução da pneumonia, gerando uma diminuição da colonização da cavidade oral, que é uma via para o pulmão (BAHRANI-MOUGEOT et al., 2007; MÜLLER, 2015; TAKAHAMA JR et al., 2020). Além disso, o custo da utilização desse produto é considerado baixo comparado ao custo de uma infecção hospitalar (GAETTI-JARDIM et al., 2013).

As doenças crônicas caracterizam uma população com prolongada permanência no hospital, intenso sofrimento, alta taxa de mortalidade e substancial consumo de recursos (LOSS et al., 2017). Buscar alternativas, desenvolver protocolos e estratégias para melhorar a recuperação do paciente, e além disso, gerenciar os recursos disponíveis para pacientes críticos, é fundamental no ambiente hospitalar. Entre as enfermidades crônicas apresentadas 
A atuação da odontologia hospitalar em uma unidade cardiovascular intensiva

nesse ambiente, comumente estão presente doenças respiratórias, condições coronárias avançadas, debilidade renal, doenças cardiovasculares, artrite e distúrbios emocionais ou psicológicos como ansiedade ou depressão (COLL et al., 2020). A doença cardíaca tem grande impacto na saúde pública, por ser comum entre os idosos e aumentar o risco de contrair pneumonia em aproximadamente duas vezes, também aumentando o risco de morte relacionado a ela (GOMES; ESTEVES, 2012a).

Portanto, objetivo deste estudo é apresentar a atuação de uma equipe odontológica em uma Unidade de Cardiologia Intensiva (UCI); uma subunidade de uma Unidade de Terapia Intensiva (UTI) de um Hospital Universitário; considerando que há uma série de ações preventivas, diagnósticas, terapêuticas e paliativas em saúde bucal que podem influenciar na saúde sistêmica e na qualidade de vida dos pacientes.

\section{MÉTODOS}

Trata-se de um artigo enfocando atuações, atividades e procedimentos realizados por uma equipe odontológica composta por estudantes de graduação e uma residente multiprofissional Cirurgiã Dentista sob supervisão de uma professora do curso de Odontologia da Universidade Federal de Santa Maria. Esta experiência ocorreu durante os meses de janeiro de 2015 e dezembro 2017, na Unidade Cardiovascular Intensiva do Hospital Universitário de Santa Maria. No total foram beneficiados 312 pacientes com os atendimentos odontológicos, sendo a média de idade desses pacientes de 63 anos.

O projeto de pesquisa foi submetido ao Comitê de Ética em Pesquisa (CEP) da Universidade Federal de Santa Maria (UFSM), obtendo parecer favorável conforme o Certificado de Apresentação para Apreciação Ética (CAAE) n.55285416.5.0000.5346. Foram seguidas as recomendações das Diretrizes e Normas da Pesquisa envolvendo Seres Humanos, apresentadas na Resolução 466/2012 do Conselho Nacional de Saúde (2012).

\section{APRESENTAÇÃO}

A Unidade Cardiovascular Intensiva é composta por seis leitos, onde são tratados pacientes com doenças cardíacas agudas ou descompensação aguda de cardiopatia crônica. A unidade é formada por uma equipe multiprofissional composta de enfermeiros, nutricionistas, técnicos em enfermagem, fonoaudiólogos, fisioterapeutas, psicólogos, médicos e cirurgiões-dentistas. 
A atuação da odontologia hospitalar em uma unidade cardiovascular intensiva

A atuação da equipe odontológica iniciou no ano de 2015, com o desenvolvimento de uma capacitação proposta pela equipe odontológica a todos os profissionais que atuavam na UCI com o objetivo de padronizar os procedimentos de higiene bucal nos leitos. $\mathrm{O}$ principal assunto abordado foi a importância da correta higienização bucal em pacientes com ventilação mecânica pré e pós cirúrgicos, a fim de colaborar para diminuir níveis de pneumonias aspirativas, conforme os estudos previamente mostram na literatura acadêmica (BAHRANI-MOUGEOT et al., 2007; MÜLLER, 2015; TAKAHAMA JR et al., 2020).

Foi proposto um protocolo com a utilização de uma técnica que preconiza a utilização do dedo envolto em uma gaze embebida em digluconato de clorexidina $0,12 \%$, com movimentos de trás para frente na cavidade bucal, no sentido de trazer os microrganismos para fora da cavidade bucal. Foi preconizada a realização de aspiração da cavidade bucal e orofaringe, no início, meio e fim do atendimento. Ligava-se o aspirador e a sonda de aspiração n.12 que era adaptada ao látex do aspirador, posicionava-se o paciente mantendo cabeceira elevada entorno de $30 \square$ a $45 \square$, quando não havia contraindicação do posicionamento pela equipe multidisciplinar, abaixava-se o siderail do lado de trabalho, e após assegurar-se corretamente sobre a fixação do tubo orotraqueala aspiração era realizada. Nos casos em que havia contraindicação sobre o posicionamento do paciente, o procedimento era discutido com a equipe multiprofissional e avaliado se iria ser feito ou não. Após realização da higiene, sugeriu-se a aplicação de saliva artificial nos pacientes com quadros de xerostomia e aplicação Dexpantenol50mg/g em fissuras e rachaduras provenientes de ressecamento labial. Dessa forma, preconizou-se realizar esse protocolo em três turnos diários após as alimentações.

Com a padronização do procedimento de higiene bucal, a atuação da equipe de Odontologia se propôs estar durante três vezes na semana a realizar a higiene dos pacientes e, diariamente, em três turnos, ela deveria ser realizada pelos técnicos de enfermagem. Além do acompanhamento da higiene bucal, foram avaliadas lesões em cavidade bucal, biofilme e lesões cariosas. Os pacientes também foram acompanhados de forma sistêmica pela equipe odontológica em conjunto com a equipe multiprofissional da UCI.

Com relação à avaliação da cavidade bucal, a candidíase ou candidose foi a infecção fúngica mais encontrada nos pacientes, sendo confirmada através das raspagens das lesões descolando a placa e visualizando uma base eritematosa acompanhados dos sinais e sintomas associados à história médica e odontológica. Neste contexto, o caso clínico era discutido em equipe e elaborado um plano de tratamento sempre considerando a situação sistêmica do paciente,todos os casos foram resolvidos com a prescrição de antifúngicos.Em alguns casos, 
A atuação da odontologia hospitalar em uma unidade cardiovascular intensiva

quando o exame clínico constatava necessidade, foram realizados procedimentos restauradores atraumáticos, com a utilização de instrumentos manuais e com o uso do cimento de ionômero de vidro (CIV), aplicado para o selamento de cicatrículas e fissuras em risco de cárie.Além disso, o CIV era utilizado pararestauração de dentes com cavidades, com o objetivo de evitar o avanço das lesõesde cárie e impedir possíveis infecções e quadros dolorosos.

Também, foram realizadas raspagens supragengivais com o uso de curetas periodontais, com a finalidade de remover cálculos.Entretanto, nos casos mais complexos como abcessos agudos e pulpites, preconizou-se que esses seriam discutidos entre a equipe multiprofisssional e os riscos da realização de procedimentos invasivos, como exodontias. Tratamentos endodônticos seriam avaliados, considerando as condições sistêmicas do paciente: se fosse de comum acordo com a equipe e não houvesse riscos de piora das condições sistêmicas, os procedimentos eram realizados no leito do paciente.Uma limitação deste relato de experiência é não ter o número exato dos procedimentos específicos para cada procedimento odontológico realizado.

\section{DISCUSSÃO}

Esse estudo tem o objetivo de apresentar as ações de uma equipe odontológica atuando em uma Unidade de Cardiologia Intensiva (UCI). Com o avanço da idade, o sistema cardiovascular passa por uma série de alterações, tais como arterioesclerose, diminuição da dispensabilidade da aorta e das grandes artérias, comprometimento da condução cardíaca e redução na função barorreceptora, que são mecanorreceptores relacionados à regulação da pressão arterial.

Observou-se que a maioria dos pacientes que são atendidos na UCI está na faixa etária de idosos. As estatísticas mostram que a maior causa de mortalidade e morbidade em idosos é a doença cardiovascular (ZASLAVSKY; GUS, 2002)e, além disso, idade maior que 60 anos também foi referida em outro estudo como uma variável independente associada ao óbito (LISBOA et al., 2007). Sabe-se que a pneumonia está frequentemente associada à insuficiência cardíaca, sendo que o aumento do fluido alveolar pulmonar e alterações nos mecanismos de defesa locais levam a este risco aumentado (GOMES, 2001).

Pacientes idosos apresentam alterações nas barreiras de defesa das mucosas, tornando-os mais suscetíveis à colonização da orofaringe (COLL et al., 2020; MÜLLER, 2015). Além disso, os pacientes internados em UTI estão expostos a diversos outros fatores 
A atuação da odontologia hospitalar em uma unidade cardiovascular intensiva

de risco, como a diminuição da limpeza natural da boca promovida pela mastigação de alimentos duros e fibrosos e a movimentação da língua e das bochechas durante a fala. Há também a redução do fluxo salivar causando xerostomia pela utilização de alguns medicamentos, que contribuem para o aumento do biofilme e favorecem a colonização oral por patógenos respiratórios (COLL et al., 2020; MÜLLER, 2015).

No que se refere à procedência dos pacientes deste estudo, em sua maioria, são originários de outras Unidades de Saúde e Pronto-Atendimentos, onde muitas vezes passam por intervenções e cuidados em unidades com estrutura de complexidade intermediária, sem materiais e equipamentos para realização do cuidado ao paciente em estado crítico (LISBOA et al., 2007). No período de atuação da equipe de Odontologia na UCI foram realizados procedimentos odontológicos com a finalidade de melhorar a qualidade de vida e o quadro sistêmico do paciente. Frequentemente foram diagnosticados casos de candidíase oral, lesões ulceradas e traumatismo de tecidos moles que foram tratados no próprio leito dos pacientes. Diagnósticos que necessitavam de imagens foram auxiliados por tomografia computadorizada CtdoBem (RENZ et al., 2018). Também foram tratadas doença periodontal e cárie nas situações em que a equipe da UCI concluía, junto à odontologia, que era importante para a recuperação geral do paciente, realizando assim o tratamento restaurador atraumático (ART) e tratamento periodontal.

A maioria dos pacientes deste relato fazia uso de próteses dentárias totais ou parciais que, quando desadaptadas, mal higienizadas ou usadas de forma contínua (sem remover para que a mucosa ficasse livre por um período de tempo diário, como para dormir à noite) podem estar relacionadas à xerostomia, ocasionando lesões orais (TORRES et al., 2002). É de extrema dificuldade promover a qualidade de vida em um ambiente onde a preocupação mais eminente é a luta contra a morte (ARAÚJO et al., 2009). Uma adequada avaliação odontológica pode determinar a necessidade e o tempo apropriados para intervir em situações de riscos futuros de contaminação, e a adequação bucal pode transformar o desfecho clínico, reduzindo fatores que possam influenciar negativamente o tratamento sistêmico e melhorar o bem-estar do paciente, dando condições mais dignas de atendimento (GOMES; ESTEVES, 2012).

Além disso, os cuidados com a higiene oral muitas vezes não são considerados prioritários, e talvez sua frequência tenha sido menor do que a necessária, tendo em vista que não houve um controle para verificar se a higiene estava sendo realizada pelos demais profissionais da maneira como foi abordada na capacitação. Entretanto, é importante ressaltar que além do benefício no bem-estar, a atenção odontológica com a elaboração de 
A atuação da odontologia hospitalar em uma unidade cardiovascular intensiva

protocolos preventivos possui custos muito baixos, podendo chegar a $10 \%$ do custo investido no manejo dos pacientes com pneumonia aspirativa instalada (PAJU; SCANNAPIECO, 2007; ROSENTHAL, 2006).

\section{CONCLUSÃO}

É possível concluir que a presença de Cirurgiões Dentistas em ambiente hospitalar é imprescindível, considerando que há uma série de ações preventivas, diagnósticas, terapêuticas e paliativas em saúde bucal que podem influenciar a saúde sistêmica e a qualidade de vida dos pacientes. Assim sendo, tenta-se controlar a presença e o aumento da proliferação de fungos e bactérias e, consequentemente, infecções e doenças sistêmicas que representam risco para a saúde do paciente, principalmente a infecção nosocomial, promovendo o bem-estar do paciente. Portanto, é importante que a prática odontológica vire rotina no ambiente hospitalar, inclusive no atendimento dos pacientes internados em Unidades Cardiovasculares Intensivas. Sugere-se que estudos transversais e de coorte sejam realizados para colaborar e fomentar políticas de saúde que apoiem a Odontologia Hospitalar.

\section{REFERÊNCIAS:}

AMARAL, S. M.; CORTÊS, A. DE Q.; PIRES, F. R. Pneumonia nosocomial: importância do microambiente oral. Jornal Brasileiro de Pneumologia, v. 35, n. 11, p. 1116-1124, nov. 2009.

ARAÚJO, R. J. G. DE et al. Análise de percepções e ações de cuidados bucais realizados por equipes de enfermagem em unidades de tratamento intensivo. Revista Brasileira de

Terapia Intensiva, v. 21, n. 1, mar. 2009.

BAHRANI-MOUGEOT, F. K. et al. Molecular Analysis of Oral and Respiratory Bacterial Species Associated with Ventilator-Associated Pneumonia. Journal of Clinical Microbiology, v. 45, n. 5, p. 1588-1593, 1 maio 2007.

COLL, P. P. et al. The Prevention of Infections in Older Adults: Oral Health. Journal of the American Geriatrics Society, v. 68, n. 2, p. 411-416, fev. 2020.

GAETTI-JARDIM, E. et al. Atenção Odontológica a Pacientes Hospitalizados: Revisão da Literatura e Proposta de Protocolo de Higiene Oral. Revista Brasileira de Ciências da Saúde - USCS, v. 11, n. 35, 20 maio 2013.

GOMES, L. Fatores de risco e medidas profiláticas nas pneumonias adquiridas na comunidade. Jornal de Pneumologia, v. 27, n. 2, p. 97-114, mar. 2001. 
A atuação da odontologia hospitalar em uma unidade cardiovascular intensiva

GOMES, S. F.; ESTEVES, M. C. L. Atuação do cirurgião-dentista na UTI: um novo paradigma. Rev. bras. odontol., v. 69, n. 1, p. 4, 2012a.

GOMES, S. F.; ESTEVES, M. C. L. Atuação do cirurgião-dentista na UTI: um novo paradigma. Rev. bras. odontol., v. 69, n. 1, p. 4, 2012 b.

LISBOA, T. et al. Prevalência de infecção nosocomial em Unidades de Terapia Intensiva do Rio Grande do Sul. Revista Brasileira de Terapia Intensiva, v. 19, n. 4, p. 414-420, dez. 2007.

LOSS, S. H. et al. Chronic critical illness: are we saving patients or creating victims? Revista Brasileira de Terapia Intensiva, v. 29, n. 1, 2017.

MÜLLER, F. Oral Hygiene Reduces the Mortality from Aspiration Pneumonia in Frail Elders. Journal of Dental Research, v. 94, n. 3_suppl, p. 14S-16S, mar. 2015.

PAJU, S.; SCANNAPIECO, F. Oral biofilms, periodontitis, and pulmonary infections. Oral Diseases, v. 13, n. 6, p. 508-512, nov. 2007.

RENZ, J. P. et al. Tomografia computadorizada multidetectores de baixa dose de radiação para avaliação de canino incluso. Revista da Faculdade de Odontologia - UPF, v. 23, n. 3, p. 291-299, 18 dez. 2018.

ROSENTHAL, V. D. Device-Associated Nosocomial Infections in 55 Intensive Care Units of 8 Developing Countries. Annals of Internal Medicine, v. 145, n. 8, p. 582, 17 out. 2006.

TAKAHAMA JR, A. et al. Analysis of oral risk factors for ventilator-associated pneumonia in critically ill patients. Clinical Oral Investigations, 27 jun. 2020.

TERPENNING, M. Geriatric Oral Health and Pneumonia Risk. Clinical Infectious Diseases, v. 40, n. 12, p. 1807-1810, 15 jun. 2005.

TORRES, S. R. et al. Relationship between salivary flow rates and Candida counts in subjects with xerostomia. Oral Surgery, Oral Medicine, Oral Pathology, Oral Radiology, and Endodontology, v. 93, n. 2, p. 149-154, fev. 2002.

ZASLAVSKY, C.; GUS, I. Idoso: Doença Cardíaca e Comorbidades. Arquivos Brasileiros de Cardiologia, v. 79, n. 6, dez. 2002.

Recebido em: 11/03/2020

Aceito em: 24/07/2020 